\title{
Flotillins control zebrafish epiboly through their role in cadherin-mediated
}

\author{
cell-cell adhesion
}

Eduardo A. Rios Morris ${ }^{1-3}$, Stéphane Bodin ${ }^{1}$, Bénédicte Delaval ${ }^{1}$, Franck Comunale ${ }^{1}$, Virginie Georget $^{1-2}$, Manoel L. Costa ${ }^{3}$, Georges Lutfalla ${ }^{4}$, Cécile Gauthier-Rouvière ${ }^{1 *}$

${ }^{1}$ CRBM, CNRS, UMR 5237, Université de Montpellier, Equipe Labellisée Ligue contre le Cancer, 1919 Route de Mende, 34293 Montpellier, France.

${ }^{2}$ MRI-CRBM Imaging facility, CNRS, UMR 5237, Université de Montpellier, 1919 Route de Mende, 34293 Montpellier, France.

${ }^{3}$ Laboratório de Diferenciação Muscular e Citoesqueleto, Departamento de Histologia e Embriologia, Instituto de Ciências Biomédicas, Universidade Federal do Rio de Janeiro, Rio de Janeiro, RJ 21949590, Brazil

${ }^{4}$ Laboratoire de Dynamique des Interactions Membranaires Normales et Pathologiques, Université de Montpellier II et I, CNRS, UMR 5235, Place Eugène Bataillon, 34095 Montpellier, France

\section{Corresponding author: cecile.gauthier@crbm.cnrs.fr}

Running Head: Flotillins are required for epiboly in zebrafish

This article has been accepted for publication and undergone full peer review but has not been through the copyediting, typesetting, pagination and proofreading process, which may lead to differences between this version and the Version of Record. Please cite this article as doi: 10.1111/boc.201700001.

This article is protected by copyright. All rights reserved. 


\begin{abstract}
Zebrafish gastrulation and particularly epiboly that involves coordinated movements of several cell layers is a dynamic process for which regulators remain to be identified. We show here that Flotillin 1 and 2, ubiquitous and highly conserved proteins, are required for epiboly. Flotillins knockdown compromised embryo survival, strongly delayed epiboly and impaired deep cell radial intercalation and directed collective migration without affecting enveloping layer cell movement. At the molecular level, we identified that Flotillins are required for the formation of E-cadherin-mediated cell-cell junctions. These results provide the first in vivo evidence that Flotillins regulate E-cadherin-mediated cell-cell junctions to allow epiboly progression.
\end{abstract}

\title{
Key words: Zebrafish, Gastrulation, Flotillins, E-cadherin
}

\section{Introduction}

Cell migration and tissue organization in development and disease are regulated by complexes networks. Cell adhesion is essential for these processes and E-cadherin-mediated cell-cell adhesion plays a pivotal role in morphogenesis and metastasis formation (Takeichi, 2011). Gastrulation is at the heart of animal development and depends on morphogenetic processes that involve collective cellular movement, while maintaining cell-cell adhesion; therefore, it has emerged as an established system to study the regulation of cell adhesion (Arboleda-Estudillo et al, 2010; Rohde \& Heisenberg, 2007). Epiboly is an important morphogenetic movement during gastrulation. It starts at the end of the

This article is protected by copyright. All rights reserved. 
blastula period and consists in the spreading of blastodermal cells over the yolk cell, moving from the animal to the vegetal pole (Lepage \& Bruce, 2010; Warga \& Kimmel, 1990). At this stage, three different cell populations exist: the yolk syncytial layer (YSL), the enveloping layer (EVL), and the deep cells (DCs). The YSL is a yolk-free cytoplasmic region of the yolk cell that is formed under the blastula cells before gastrulation starts (Carvalho \& Heisenberg, 2010). The YSL provides forces through an actin-myosin ring that surrounds the gastrula and contracts to pull the YSL to the vegetal pole and also through oriented bundles of microtubules that pull YSL nuclei toward the vegetal pole (Behrndt et al, 2012; Cheng et al, 2004). The EVL, the most external single-cell-thick layer that covers the mass of DCs, is attached to the YSL by tight junctions allowing the EVL to be pulled, together with the YSL, to the vegetal pole (Koppen et al, 2006; Siddiqui et al, 2010). DCs consist of several layers of cells that start to spread over the yolk and intercalate to form a very thin layer at the end of gastrulation, a process called radial cell intercalation (Kane et al, 2005; Lepage \& Bruce, 2010). Proper epiboly involves coordinated movements of all these layers. Radial cell intercalation and mesendoderm collective migration are controlled by several pathways, among which the cell-cell adhesion protein E-cadherin (Arboleda-Estudillo et al, 2010; Lepage \& Bruce, 2010). E-cadherin is the first classical cadherin expressed at the onset of zebrafish gastrulation, and it is required for mesendodermal cell cohesion and migration. In E-cadherin mutant embryos and morphants, DC movement and migration are perturbed, whereas the YSL and EVL can migrate to the vegetal pole, leaving behind the DCs. This DC movement defect is due to impaired radial intercalation (Kane et al, 2005; McFarland et al, 2005; Montero et al, 2005; Shimizu et al, 2005).

Flotillins were recently identified as regulators of cadherin complex dynamics at the plasma membrane (Guillaume et al, 2013; Kurrle et al, 2013). Flotillin 1 and 2 are ubiquitous and highly conserved proteins. They localize at the cytoplasmic face and in specific cholesterol-rich membrane microdomains of the plasma membrane and in intracellular vesicles. Studies conducted in different cell lines showed that Flotillins are involved in vesicle trafficking and endocytosis (Ait-Slimane et al, 
2009; Glebov et al, 2006), cell adhesion (Guillaume et al, 2013; Kurrle et al, 2013; Solis et al, 2013), regulation of the actin cytoskeleton (Neumann-Giesen et al, 2007) in EGFR signaling (Amaddii et al, 2012) and morphogen secretion (Katanaev et al, 2008).

We decided to use the zebrafish gastrulation as an in vivo model to analyze the role of Flotillins in cellular migration. In zebrafish, three Flotillin isoforms, Flotillin 1a, Flotillin $1 \mathrm{~b}$ and Flotillin 2a, are expressed from the one-cell stage throughout early development (von Philipsborn et al, 2005). Through specific morpholino injections, we assessed the role of Flotillins during gastrulation. We demonstrated that Flotillins are required for epiboly and showed that they play critical roles in the regulation of E-cadherin-mediated cell-cell adhesion and hence in DC radial intercalation and directional movement during zebrafish epiboly progression.

\section{Results and discussion}

To assess the role of Flotillin 2 during zebrafish gastrulation, its expression was knocked-down using two different anti-sense morpholino oligonucleotides (Fig. 1A, S1 and S2). MoATGFlot2 morpholino oligonucleotide recognizes the ATG starting codon and inhibits translation of the maternal and zygotic of Flotillin 2 mRNAs. In MoATGFlot2-injected embryos, the expression of Flotillin 2 at 75\% epiboly was reduced, supporting the efficiency of this morpholino to downregulate Flotillin 2 expression. MoSplSFlot2 morpholino oligonucleotide targets the intron 2-exon 3 junction and disrupts the splicing, and hence translation of only the zygotic Flotillin 2 mRNA. For this reason, Flotillin 2 expression was also decreased in MoSplSFlot2 morphants (MO), but to a lesser extent compared with MoATGFlot2 MO embryos (Fig. 1A). As previously reported, decreasing the amount of Flotillin 2 also led to a reduction of Flotillin 1 expression in both MoATGFlot2 and MoSplSFlot2 MOs (Guillaume et al, 2013; Vassilieva et al, 2009). This indicates that the targeting of Flotillin 2 caused 
the concomitant decrease of Flotillin 1 expression, which led us to talk about Flotillins knock down. Because the MoATGFlot2 is more efficient in knocking down Flotillins, we mainly used this morpholino for the functional analysis. Results were compared with the phenotype obtained using the standard control morpholino (Gene Tools, LLC) (MoCtrl).

Upon MoATGFlot2 injection at the 1-cell stage, MO embryos showed cell organization defects from the 16-cells stage and at least until the 1000-cells stage (Fig. 1B). At the dome stage, no major morphological difference was observed compared with embryo injected with control morpholino. However, Flotillins MO embryos showed major epiboly defects including a strong delay or a complete arrest of epiboly progression with a high level of penetrance (Fig. 1C). Embryos arrested at early gastrula stages did not complete gastrulation and were discarded; only embryos that reached $75 \%$ epiboly were further analyzed. Re-expression of a mouse Flotillin 2 mRNA partially rescued this epiboly arrest and more than 50\% of injected embryos developed normally (Fig. 1B,C). Similar epiboly defects (Fig. 1C) were observed in MoSplSFlot2 Mo or using a mutant in which Flotillin 2 was knocked out using the CRISPR/Cas9 technology (CRIPR/Cas9 Flot2; see Fig. S1Fa-c for its characterization). These early major defects in Flotillins MO embryos are in agreement with Flotillins expression in early zebrafish development (von Philipsborn et al, 2005). We analyzed Flotillin 1 and 2 expression during gastrulation (from the 4-cells stage) by immunofluorescence microscopy of wholemount embryos and confirmed that they are expressed at 4-, 16- and 64-cells stage (Fig. 1D). These data show that Flotillins are expressed and localized at cell-cell junctions during the blastula stage and that they are required during gastrulation and more specifically for epiboly. Analysis of the expression of mesodermal markers and organizer-specific genes (Fig. S3) indicated that Flotillins are not involved in mesodermal cell fate specification and early patterning, but Flotillins disruption affects cell organization. In addition, Flotillins knock-down generated a series of hypomorphic phenotypes due to variable reduction (Mo efficiency or variations in kinetics due to maternal expression) in Flotillins protein expression. MO embryos that completed epiboly presented morphological alterations

This article is protected by copyright. All rights reserved. 
at post-gastrulation stages, such as shorter antero-posterior axis (obvious at $16 \mathrm{hpf}$ ) and stunted tail (at 24 hpf) (Fig. 1B), indicating defects in convergence-extension movements, as confirmed by MyoD analysis (Fig. S3). Whereas the loss of Flotillins does not appear to perturb the viability of mouse and fly embryos (Banning et al, 2014; Hoehne et al, 2005; Katanaev et al, 2008; Ludwig et al, 2010), we show that zebrafish hardly complete epiboly in the absence of Flotillins. Zebrafish epiboly is, thus, a good model to analyze the in vivo function of Flotillins.

To identify the cellular mechanisms underlying the epiboly alterations in Flotillins MO embryos, we stained embryos at $75 \%$ epiboly with phalloidin to observe the F-actin cytoskeleton and with Hoechst to visualize cell nuclei in order to monitor epiboly progression by confocal microscopy (Fig. 2A for MoATGFlot2 and Fig. S1Ea for MoSplFlot2 embryos). In control embryos, DCs followed the EVLYSL movement to the vegetal pole. In contrast, in Flotillins MO embryos, we observed a prominent and specific defect in the ability of DC to move together with EVL cells to the vegetal pole. This was quantified by morphometric analyses to measure the distance between the DC margin and the F-actin ring in YSL at the EVL margin (Fig. 2B). We confirmed this DC migration defect in CRIPR/Cas9 Flot2 embryos (Fig. S1Fd). DC inability to follow EVL-YSL migrating cells could be explained by defects in the radial cell intercalation movements of DCs during epiboly (Arboleda-Estudillo et al, 2010; Schepis et al, 2012). Thus, we performed time-lapse confocal microscopy and individual DC tracking to analyze and quantify cell movement and coordination (Fig. 2C-G). In control embryos, DCs moved from the deepest layers to the surface, progressing from several layers to one thin layer at the end of the epiboly (Fig. 2C and corresponding supplemental video 1, see the yellow colored cell marked with a star). In contrast, in Flotillins MO embryos, DCs followed a process called reverse radial intercalation in which they appear in the superficial epiblast layer and then move back into the deeper layers (Fig. 2C for MoATGFlot2 embryos and corresponding supplemental video 1, see the appearing and disappearing yellow colored cell marked with a star and Fig. S1Eb for MoSplFlot2 embryos). The DC trajectories in control embryo were characteristic of a directional collective 
movement, whereas DC in Flotillins MO embryos appeared perturbed and less coordinated (Fig. 2D). Whereas the velocity was not significantly different in DCs from control and Flotillins MO (data not shown), DC directionality in Flotillins MO was affected, compared with control embryos (Fig. 2E). Quantification of cell-cell contact duration revealed that in Flotillins MO embryos, DC time of contact was decreased compared with control embryos (Fig. 2F).

To study in more details the dynamic movements of DCs during radial intercalation, we performed a quantitative 3-dimensional (3D) analysis of their trajectories. One-cell-stage embryos were injected with GFP-CAAX-Ras mRNA that is incorporated into the plasma membrane and allows following cells using time-lapse confocal microscopy. In control embryos, the DC movement displayed a clear directionality, from the deeper layer to the surface, where they intercalated between other cells of the outer layer and became stabilized at the end of gastrulation (Fig. 2G and corresponding supplemental movie 2). In Flotillins MO embryos, DCs also appeared from the deeper layers, but did not stay in the outer cell layer and moved back. A quantitative analysis of the DC 3D movements indicated that the instantaneous speed and total speed (Fig. 2L) were not significantly different, whereas the net speed and the persistence of migration of DCs were significantly different between controls and Flotillins MO embryos (Fig. 2H-K).

Altogether, these data indicate that the knock-down of Flotillins strongly affects DC radial cell intercalation movement. In contrast, EVL cell migration during epiboly is not affected in Flotillins MO embryos. This Flotillins MO phenotype is very similar to the phenotype of E-cadherin deficient embryos (Koppen et al, 2006). Interestingly, Flotillins were recently shown to stabilize cadherins at cell-cell contact sites in various cell lines (Guillaume et al, 2013; Kurrle et al, 2013) and we detected Flotillins accumulation at cell-cell contacts in embryos from the 4-cell-stage until mid-gastrula (Fig. 1D and Fig. S2A). We thus analyzed Flotillins and E-cadherin co-distribution in zebrafish embryos and checked whether Flotillins depletion also affected the cadherin complex in DCs. Immunofluorescence analysis of E-cadherin expression in Flotillin 2-GFP-expressing embryos at 75\% 
epiboly showed that Flotillin 2 and E-cadherin co-localized at cell-cell contacts of DCs, where they also co-localized with F-actin (Fig. 3A). Many studies have reported that Flotillins are localized at cell-cell contact sites in different cell types in culture (Babuke et al, 2009; Fernow et al, 2007; Liu et al, 2005; Malaga-Trillo et al, 2009; Morrow et al, 2002; Solis et al, 2007; Stuermer et al, 2001) and in vivo in neural cells (von Philipsborn et al, 2005), which is in agreement with their role in axon regeneration (Munderloh et al, 2009; Schulte et al, 1997). Here, we demonstrated that the Flotillins/Ecadherin co-localization occurs very early and is maintained during gastrulation.

Next, we analyzed whether Flotillins knock-down affected the distribution of E-cadherin and its direct partner $\beta$-catenin in DCs. In embryos injected with MoCtrl, E-cadherin and $\beta$-catenin accumulated at cell-cell contacts of DCs. In contrast, in Flotillins MO we observed a marked decrease of E-cadherin and $\beta$-catenin accumulation at cell-cell contacts of DCs (Fig. 3B, C and D). Line scanning of the signal at cell-cell contacts was used for quantification of the ratio of the signal at cell-cell contacts (CCC) to the signal outside cell-cell contacts (NCC) (Fig. 3F, G and H). These data indicated that Flotillins knock-down affected the accumulation of E-cadherin and $\beta$-catenin at cell-cell contacts, whereas F-actin signal was not significantly reduced (Fig. 3E for MoATGFlot2, Fig.1Ec-d for MoSplFlot2 and Fig. 1Fe-f for CRISPR/Cas9 Flot2 embryos). We also assessed the effects of Flotillins knock-down on the total amount of E-cadherin and $\beta$-catenin proteins in injected embryos at $75 \%$ epiboly. In contrast to $\beta$-catenin, the total amount of E-cadherin protein was strongly decreased in Flotillins MO embryos (Fig. 3I). Treatment of Flotillins MO embryos with proteasomal or lysosomal inhibitors (MG-132 and chloroquine, respectively) stabilized E-cadherin but we were unable to detect E-cadherin accumulation at cell-cell junctions (data not shown). These data indicate that Flotillins are required for the stabilization of E-cadherin and $\beta$-catenin at cell-cell contact sites of DCs. Moreover, the absence of Flotillins is associated with a decrease in the amount of E-cadherin protein. N-cadherin is also expressed during gastrulation but we were not able to analyze its distribution due to absence of functional antibodies. Several studies reported that the perturbation of 
cadherin-mediated cell-cell adhesion impairs gastrulation movements and particularly epiboly (Babb \& Marrs, 2004; Kane et al, 2005; Schepis et al, 2012; Shimizu et al, 2005; Tay et al, 2010; Ulrich et al, 2005). Specifically, E-cadherin-deficient embryos display reduced radial intercalation movement of DCs, while EVL cells complete epiboly, indicating that DCs use E-cadherin-mediated cell-cell adhesion between them and/or the EVL substrate during epiboly progression (Kane et al, 2005; Shimizu et al, 2005). Recently, it has emerged that different mechanisms that affect E-cadherin function also perturb epiboly, such as the regulation of proper plasma membrane localization of Ecadherin endocytosis by WNT11 through RAB5 (Ulrich et al, 2005), the prion protein that relocalizes E-cadherin into RAB11-positive vesicles (Malaga-Trillo et al, 2009), the Rho GTPase CHP that allows E-cadherin stabilization at the plasma membrane through PAK and $\beta$-PIX (Tay et al, 2010), or POU5F1/OCT4 that acts through EGFR signaling (Song et al, 2013). Other pathways affect Ecadherin differently. For instance, $\mathrm{G \alpha}_{12 / 13}$ inhibits E-cadherin activity through direct binding to its intracellular domain (Lin et al, 2009), whereas 2-O-sulfotransferase induces, though heparan sulfate proteoglycans, GSK3-mediated $\beta$-catenin degradation (Cadwalader et al, 2012). Finally, because Flotillin 1 was shown to regulate Ephrin B2 protein level by tuning its degradation by ADAM10, a protease that also clives E-cadherin (Ji et al, 2014; Solanas et al, 2011), we tested the effect of GM6001, a broad-spectrum metalloprotease inhibitor. Whereas this treatment allowed some Ecadherin stabilization (Fig. 3J), this was not enough to rescue the Flotillins MO embryo phenotype, suggesting the existence of several additional mechanisms.

The phenotype of the Flotillins MO embryos, with the perturbation of DC movement, their radial intercalation and the associated defects in epiboly, whereas EVL cell migration during epiboly is not affected, is in complete agreement with a role of Flotillins in cadherin-mediated adhesion (Fig. 4). Interestingly, in EVL cells of Flotillins MO embryos, E-cadherin-mediated cell-cell adhesion is affected. This indicates that other adhesion complexes, such as EpCAM or tight junctions, could compensate (Siddiqui et al, 2010; Slanchev et al, 2009). Interestingly, we showed that the expression

This article is protected by copyright. All rights reserved. 
and localization of the tight junction-associated scaffold protein ZO-1 is not perturbed in Flotillins MO embryos (Fig. S2C). Moreover, E-cadherin is not accumulated at the EVL-to-YSL interface that plays an important role during EVL epiboly. The analysis of this region by transmission electron microscopy revealed characteristics of tight junctions and not of adherens junction (Koppen et al, 2006). Moreover, F-actin accumulation within the YSL along the margin of the enveloping layer is not altered in Flotillins MO embryos, suggesting that the contractile forces required for the spreading of EVL cells over the yolk are not affected (Behrndt et al, 2012; Koppen et al, 2006).

In summary, our work revealed that, in zebrafish, Flotillins are required for the formation of Ecadherin-mediated cell-cell junctions in vivo and, as a consequence, for the regulation of the DC radial cell intercalation process and directed collective DC migration during epiboly. Therefore, we identified Flotillins as novel actors of epiboly in zebrafish.

\section{Experimental procedures}

\section{Zebrafish strains}

Zebrafish (Danio rerio) AB lines were raised and kept under standard laboratory conditions (Westerfield, 2000) according to European Union guidelines for the handling of laboratory animals (http://ec.europa.eu/environment/chemicals/lab_animals/home_en.htm) and were approved by the Direction Sanitaire et Vétérinaire de l'Hérault and Comité d'Ethique pour l'Expérimentation Animale under reference CEEA-LR-13007.

Morpholino oligonucleotides, RNA synthesis and microinjection

This article is protected by copyright. All rights reserved. 
Two morpholino (Mo) oligonucleotides targeting Flotillin 2 mRNA were designed; MoATGFlot2 (5'ACCGTGTAGCAATTCCCCATTTCC-3') and MoSplFlot2 (5'-tccacag GATAACCCTTGAGA-3'). Standard oligonucleotide from Gene Tools Inc. (5'-CCTCTTACCTCAGTTACAATTTATA-3') was used as a control morpholino. The expression vectors pCS2+Flotillin2-EGFP, pCS2+Flotillin2mCherry and pCS2+E-cadherin-EGFP were linearized with NotI and taken as templates to synthesize capped mRNA using mMessage Machine ${ }^{\circledR}$ SP6 in vitro transcription kit (Ambion ${ }^{\circledR}$ - Thermo Fisher). The synthesis was done according to the manufacturer's protocol and then purified by phenol:chlorophorm extraction and isopropanol precipitation. The concentration was determined by Nanodrop. Single cell stage embryos were injected either with Mo (8 ng per embryo) or mRNA (300 pg per embryo), incubated at $28^{\circ} \mathrm{C}$ and examined at mid-gastrula stage.

The pCS2+Flotillin2ATG:EGFP was made by inserting the ATG region of the Flotillin 2 gene (nucleotide -169 from the ATG to nucleotide +27) upstream of EGFP in the pCS2+EGFP vector.

\section{CRISPR/Cas2 Flotillin 2 knock-out mutant}

The single guide RNA sequence (sgRNA) was designed in silico using the CRISPR design tool (http://crispr.mit.edu) that allows searching and ranking the potential target sequences ending with the NGG motif. We chose the 5'-GGTAGGACCGAACGAAGCCC-3' sequence that was ranked as first and is located in exon 1 of the flotillin 2 gene (Figure S1Fa). For in vitro transcription of the sgRNA, the DNA template was generated using the two-oligo technique (Shah et al, 2015). Two oligos were designed: a 81 nt scaffold oligonucleotide necessary for Cas9 recognition and attachment $\left(5^{\prime}\right.$ GATCCGCACCGACTCGGTGCCACTTTTTCAAGTTGATAACGGACTAGCCTTATTTTAACTT GCTATTTCTAGCTCTAAAAC-3 ' $)$, and a second $60 \mathrm{nt}$ oligonucleotide $\left(5^{\prime}\right.$ AATTAATACGACTCACTATAGGTAGGACCGAACGAAGCCCGTTTTAGAGCTAGAAATA GC-3') that contains an upstream T7 binding site sequence (required for in vitro transcription) 
followed by the Flotillin 2 target sequence and by a 20nt sequence complementary to the scaffold oligonucleotide to allow the hybridization of the two oligonuclotides for PCR amplification. After annealing and PCR amplification (performed as described by Shah, et al. 2015), the double-strand fragment $(5$

\section{AATTAATACGACTCACTATAGGTAGGACCGAACGAAGCCCGTTTTAGAGCTAGAAATA} GCAAGTTAAAATAAGGCTAGTCCGTTATCAACTTGAAAAAGTGGCACCGAGTCGGTGCG

GATC-3' ) was purified and used for in vitro transcription to generate Flotillin 2-sgRNA. In parallel, pCS2-nCas9n (gift from Wenbiao Chen) (Addgene plasmid \# 47929) (Jao et al, 2013) was linearized (with $\mathrm{XbaI}$ ) and purified and then used for in vitro transcription to generate the Cas9 mRNA.

The Cas9 mRNA and Flotillin 2-sgRNA were mixed (1200 ng/ $\mu \mathrm{l}$ and $200 \mathrm{ng} / \mu \mathrm{l}$, respective final concentration) and about $1 \mathrm{~nL}$ of the mix was injected in 1-cell embryos. As control Flotillin 2-sgRNA was injected alone. Validation of flotillin 2 knock-out after Flot2CRISPR/Cas9 injection was performed as follow. Embryos that presented any early sign of toxicity were discarded and only embryos that reached gastrula stage were harvested for analysis. To analyze the levels of Flotillin 2 mRNA, total mRNA was extracted from 20 embryos/condition, reverse transcribed and then the Flotillin 2 fragment of 424 bp was PCR-amplified.

\section{Gel electrophoresis and immunoblotting}

Injected embryos were dechorionated at shield stage with $2 \mathrm{mg} / \mathrm{ml}$ Pronase, deyolked on de-yolking buffer $\left(55 \mathrm{mM} \mathrm{NaCl}, 1.8 \mathrm{mM} \mathrm{KCl}, 1.25 \mathrm{mM} \mathrm{NaHCO}_{3}\right)$, cell dissociated in Ringer Solution containing EDTA and EGTA (116mM NaCl, 2.9mM KCl, 5mM HEPES pH7.2, 5mM EDTA 0.5mM EGTA) and lysed by trituration using Lysis buffer (10mM PIPES pH7, 100mM NaCl, 300mM Sucrose, 3mM $\mathrm{MgCl}_{2}, 0,5 \% \mathrm{NP} 40,1 \mathrm{mM}$ EDTA, $1 \mathrm{mM}$ Orthovanadate, protease inhibitor cocktail). $30 \mu \mathrm{g}$ of protein extracts were resolved on $10 \%$ polyacrylamide gels by electrophoresis and transferred to Immobilon-

This article is protected by copyright. All rights reserved. 
FL membranes (Millipore). The membranes were incubated with the indicated antibodies as described previously (Thuault et al, 2013). For protein detection and quantification, the Odyssey Infrared Imaging System (LI-COR Biosciences) was used. Immunoblots were quantified via densitometry using Odyssey V3.0 and ImageJ.

\section{Immunohistochemistry}

Mouse antibodies against Flotillin 2, E-cadherin and B-catenin (BD Biosciences, \#610383, \#610181, \#610153, respectively) were used at 1:500 dilution. The rabbit polyclonal antibodies against Flotillin 1 comes from Claudia Stuermer's laboratory (Schulte et al, 1997) and against ZO-1 from Zymed, Invitrogen, \#61730). Primary antibodies were detected with Alexa Fluor 488 or 546-conjugated goat anti-mouse or anti-rabbit IgG (Molecular Probes).

F-actin was labeled with Alexa Fluor 546 phalloidin conjugates (Molecular Probes, Interchim) and nuclei with Hoechst 33342 (Sigma-Aldrich)

Embryos were fixed in 4\% paraformaldehyde in phosphate-buffered saline (PBS) overnight at $4{ }^{\circ} \mathrm{C}$. Manually dechorionated embryos were permeabilized with $0.1 \%$ Triton X-100 (in PBS) and blocked with $5 \%$ BSA, $1 \%$ goat serum in PBS- $0.1 \%$ Triton X-100 for 4 h at RT. Primary antibodies were added and incubated overnight at $4{ }^{\circ} \mathrm{C}$. Embryos were washed three times in PBS- $0.1 \%$ Triton X-100 and incubated with Alexa Fluor 488 or 546-conjugated secondary antibodies and 546 phalloidin conjugate when needed. Finally stained embryos were soaked in $0.01 \%$ Hoechst 33342 for $1 \mathrm{~h}$ and washed five times PBS-0.1\% Triton X-100.

Images were taken using a Confocal Leica SP5-SMD with LEICA 40x/1.3 or 63x/1.4 OIL HCX PL APO CS objectives and captured with a hybrid detector (Leica HyD) controlled using the C software.

This article is protected by copyright. All rights reserved. 
Co-localization data originate from multichannel fluorescence stacks collected using a Leica SP5 microscope. Images were treated using ImageJ software (National Institutes of Health).

\section{Time-lapse imaging, Measurements of cell velocity, persistence and directionality}

Embryos were imaged using Leica SP5-SMD microscope equipped with a motorized stage, as well as a heated-regulated incubator. Nomarski and fluorescent images were taken every 4 min during 30 min, using a LEICA 10x/0.3 HCX PL objective and captured with a hybrid detector (Leica HyD) controlled using the C software, focusing on the internal layers of the DCs at the equatorial/dorsal embryonic axis at $75 \%$ epiboly. Stacks of 10 planes with a $\mathrm{Z}$ step of $1 \mu \mathrm{m}$ were taken for each time point. Manual cell tracking and " $\mathrm{x} / \mathrm{y}$ " cell position recording (of each cell at each time point) were performed using ImageJ software (Manual Tracking plugin) and the cell migration parameters were calculated using Ibidi Chemotaxis tool (http://ibidi.com/software/chemotaxis_and_migration_tool/). Data of the dynamic behavior of DCs were extracted to quantify the directionality. Time of contact was calculated following pairs of DCs throughout the experiment and extracting their contact time. For 3-D reconstruction stacks of confocal images were processed with Imaris (Bitplane, Zurich, Switzerland) and volume representations were made. 3-D cell tracking was performed to measure Total Speed, Instantaneous Speed, Net Speed and Persistence.

\section{Statistical analysis}

For experiments with $\mathrm{n}>30$, Student's $t$ test was used to assess significant differences between two experimental conditions. For experiments with $\mathrm{n}<30$, the non-parametric Mann-Whitney $\mathrm{U}$ test was used. At least three independent experiments were performed.

This article is protected by copyright. All rights reserved. 


\section{Acknowledgements}

We thank Sylvain de Rossi and Simon Lachambre from the Montpellier Imaging Facility (http://www.mri.cnrs.fr/), Caty Gonzalez, Marc Plays and Philippe Richard from the aquatic facilities, Claudia Stuermer for anti-flotillin 1 antibody.

\section{Competing interests}

No competing interests declared

\section{Author contributions}

ERM: conception, design, execution and interpretation of the data. Figures assembly; SB: conception, design and interpretation of the data; FC: design and interpretation of the data; GL: conception, design and interpretation of the data. Manuscript edition; BD: manuscript edition; VG: conception of experiments; MC: conception of MO; CGR: conception, design and interpretation of the data. Manuscript writing and figures editing.

\section{Funding}

This work was supported by the Ligue Nationale contre le Cancer (LNCC) (" Equipe labellisée »), and the Association Française contre les Myopathies (AFM), and the European Community's Seventh

This article is protected by copyright. All rights reserved. 
Framework Programme (FP7- PEOPLE-2011-ITN) under the Marie-Curie Initial Training Network FishForPharma [grant agreement no. PITN-GA-2011-289209]. C.G.R. was supported by INSERM. ERM was supported by "Ciência sem Fronteiras" program (CNPq - Brazil).

\section{References}

Ait-Slimane T, Galmes R, Trugnan G, Maurice M (2009) Basolateral internalization of GPIanchored proteins occurs via a clathrin-independent flotillin-dependent pathway in polarized hepatic cells. Molecular biology of the cell 20: 3792-3800

Amaddii M, Meister M, Banning A, Tomasovic A, Mooz J, Rajalingam K, Tikkanen R (2012) Flotillin-1/reggie-2 protein plays dual role in activation of receptor-tyrosine kinase/mitogenactivated protein kinase signaling. The Journal of biological chemistry 287: 7265-7278

Arboleda-Estudillo Y, Krieg M, Stuhmer J, Licata NA, Muller DJ, Heisenberg CP (2010) Movement directionality in collective migration of germ layer progenitors. Current biology : CB 20: 161-169

Babb SG, Marrs JA (2004) E-cadherin regulates cell movements and tissue formation in early zebrafish embryos. Developmental dynamics : an official publication of the American Association of Anatomists 230: 263-277

Babuke T, Ruonala M, Meister M, Amaddii M, Genzler C, Esposito A, Tikkanen R (2009) Heterooligomerization of reggie-1/flotillin-2 and reggie-2/flotillin-1 is required for their endocytosis. Cellular signalling 21: 1287-1297

Banning A, Regenbrecht CR, Tikkanen R (2014) Increased activity of mitogen activated protein kinase pathway in flotillin-2 knockout mouse model. Cellular signalling 26: 198-207

Behrndt M, Salbreux G, Campinho P, Hauschild R, Oswald F, Roensch J, Grill SW, Heisenberg CP (2012) Forces driving epithelial spreading in zebrafish gastrulation. Science 338: 257-260

Cadwalader EL, Condic ML, Yost HJ (2012) 2-0-sulfotransferase regulates Wnt signaling, cell adhesion and cell cycle during zebrafish epiboly. Development 139: 1296-1305

Carvalho L, Heisenberg CP (2010) The yolk syncytial layer in early zebrafish development. Trends in cell biology 20: 586-592

This article is protected by copyright. All rights reserved. 
Cheng JC, Miller AL, Webb SE (2004) Organization and function of microfilaments during late epiboly in zebrafish embryos. Developmental dynamics : an official publication of the American Association of Anatomists 231: 313-323

Fernow I, Icking A, Tikkanen R (2007) Reggie-1 and reggie-2 localize in non-caveolar rafts in epithelial cells: cellular localization is not dependent on the expression of caveolin proteins. Eur J Cell Biol 86: 345-352

Glebov 00, Bright NA, Nichols BJ (2006) Flotillin-1 defines a clathrin-independent endocytic pathway in mammalian cells. Nat Cell Biol 8: 46-54

Guillaume E, Comunale F, Do Khoa N, Planchon D, Bodin S, Gauthier-Rouviere C (2013) Flotillin microdomains stabilize cadherins at cell-cell junctions. Journal of cell science 126: 5293-5304

Hoehne M, de Couet HG, Stuermer CA, Fischbach KF (2005) Loss- and gain-of-function analysis of the lipid raft proteins Reggie/Flotillin in Drosophila: they are posttranslationally regulated, and misexpression interferes with wing and eye development. Molecular and cellular neurosciences 30: 326-338

Jao LE, Wente SR, Chen W (2013) Efficient multiplex biallelic zebrafish genome editing using a CRISPR nuclease system. Proceedings of the National Academy of Sciences of the United States of America 110: 13904-13909

Ji YJ, Hwang YS, Mood K, Cho HJ, Lee HS, Winterbottom E, Cousin H, Daar IO (2014) EphrinB2 affects apical constriction in Xenopus embryos and is regulated by ADAM10 and flotillin-1. Nature communications 5: 3516

Kane DA, McFarland KN, Warga RM (2005) Mutations in half baked/E-cadherin block cell behaviors that are necessary for teleost epiboly. Development 132: 1105-1116

This article is protected by copyright. All rights reserved. 
Katanaev VL, Solis GP, Hausmann G, Buestorf S, Katanayeva N, Schrock Y, Stuermer CA, Basler K (2008) Reggie-1/flotillin-2 promotes secretion of the long-range signalling forms of Wingless and Hedgehog in Drosophila. The EMBO journal 27: 509-521

Koppen M, Fernandez BG, Carvalho L, Jacinto A, Heisenberg CP (2006) Coordinated cell-shape changes control epithelial movement in zebrafish and Drosophila. Development 133: 2671-2681

Kurrle N, Vollner F, Eming R, Hertl M, Banning A, Tikkanen R (2013) Flotillins directly interact with gamma-catenin and regulate epithelial cell-cell adhesion. PloS one 8: e84393

Lepage SE, Bruce AE (2010) Zebrafish epiboly: mechanics and mechanisms. The International journal of developmental biology 54: 1213-1228

Lin F, Chen S, Sepich DS, Panizzi JR, Clendenon SG, Marrs JA, Hamm HE, Solnica-Krezel L (2009) Galpha12/13 regulate epiboly by inhibiting E-cadherin activity and modulating the actin cytoskeleton. The Journal of cell biology 184: 909-921

Liu J, Deyoung SM, Zhang M, Dold LH, Saltiel AR (2005) The stomatin/prohibitin/flotillin/HflK/C domain of flotillin-1 contains distinct sequences that direct plasma membrane localization and protein interactions in 3T3-L1 adipocytes. The Journal of biological chemistry 280: 16125-16134

Ludwig A, Otto GP, Riento K, Hams E, Fallon PG, Nichols BJ (2010) Flotillin microdomains interact with the cortical cytoskeleton to control uropod formation and neutrophil recruitment. The Journal of cell biology 191: 771-781

Malaga-Trillo E, Solis GP, Schrock Y, Geiss C, Luncz L, Thomanetz V, Stuermer CA (2009) Regulation of embryonic cell adhesion by the prion protein. PLoS biology 7: e55

McFarland KN, Warga RM, Kane DA (2005) Genetic locus half baked is necessary for morphogenesis of the ectoderm. Developmental dynamics : an official publication of the American Association of Anatomists 233: 390-406

This article is protected by copyright. All rights reserved. 
Montero JA, Carvalho L, Wilsch-Brauninger M, Kilian B, Mustafa C, Heisenberg CP (2005) Shield formation at the onset of zebrafish gastrulation. Development 132: 1187-1198

Morrow IC, Rea S, Martin S, Prior IA, Prohaska R, Hancock JF, James DE, Parton RG (2002) Flotillin-1/reggie-2 traffics to surface raft domains via a novel golgi-independent pathway. Identification of a novel membrane targeting domain and a role for palmitoylation. The Journal of biological chemistry 277: 48834-48841

Munderloh C, Solis GP, Bodrikov V, Jaeger FA, Wiechers M, Malaga-Trillo E, Stuermer CA (2009) Reggies/flotillins regulate retinal axon regeneration in the zebrafish optic nerve and differentiation of hippocampal and N2a neurons. J Neurosci 29: 6607-6615

Neumann-Giesen C, Fernow I, Amaddii M, Tikkanen R (2007) Role of EGF-induced tyrosine phosphorylation of reggie-1/flotillin-2 in cell spreading and signaling to the actin cytoskeleton. Journal of cell science 120: 395-406

Rohde LA, Heisenberg CP (2007) Zebrafish gastrulation: cell movements, signals, and mechanisms. International review of cytology 261: 159-192

Schepis A, Sepich D, Nelson WJ (2012) alphaE-catenin regulates cell-cell adhesion and membrane blebbing during zebrafish epiboly. Development 139: 537-546

Schulte T, Paschke KA, Laessing U, Lottspeich F, Stuermer CA (1997) Reggie-1 and reggie-2, two cell surface proteins expressed by retinal ganglion cells during axon regeneration. Development 124: $577-587$

Shah AN, Davey CF, Whitebirch AC, Miller AC, Moens CB (2015) Rapid reverse genetic screening using CRISPR in zebrafish. Nature methods 12: 535-540

This article is protected by copyright. All rights reserved. 
Shimizu T, Yabe T, Muraoka O, Yonemura S, Aramaki S, Hatta K, Bae YK, Nojima H, Hibi M (2005) E-cadherin is required for gastrulation cell movements in zebrafish. Mechanisms of development 122: $747-763$

Siddiqui M, Sheikh H, Tran C, Bruce AE (2010) The tight junction component Claudin E is required for zebrafish epiboly. Developmental dynamics : an official publication of the American Association of Anatomists 239: 715-722

Slanchev K, Carney TJ, Stemmler MP, Koschorz B, Amsterdam A, Schwarz H, Hammerschmidt M (2009) The epithelial cell adhesion molecule EpCAM is required for epithelial morphogenesis and integrity during zebrafish epiboly and skin development. PLoS genetics 5: e1000563

Solanas G, Cortina C, Sevillano M, Batlle E (2011) Cleavage of E-cadherin by ADAM10 mediates epithelial cell sorting downstream of EphB signalling. Nat Cell Biol 13: 1100-1107

Solis GP, Hoegg M, Munderloh C, Schrock Y, Malaga-Trillo E, Rivera-Milla E, Stuermer CA (2007) Reggie/flotillin proteins are organized into stable tetramers in membrane microdomains. Biochem J 403: 313-322

Solis GP, Hulsbusch N, Radon Y, Katanaev VL, Plattner H, Stuermer CA (2013) Reggies/flotillins interact with Rab11a and SNX4 at the tubulovesicular recycling compartment and function in transferrin receptor and E-cadherin trafficking. Molecular biology of the cell 24: 2689-2702

Song S, Eckerle S, Onichtchouk D, Marrs JA, Nitschke R, Driever W (2013) Pou5f1-dependent EGF expression controls E-cadherin endocytosis, cell adhesion, and zebrafish epiboly movements. Developmental cell 24: 486-501

Stuermer CA, Lang DM, Kirsch F, Wiechers M, Deininger SO, Plattner H (2001) Glycosylphosphatidyl inositol-anchored proteins and fyn kinase assemble in noncaveolar plasma membrane microdomains defined by reggie-1 and -2. Molecular biology of the cell 12: 3031-3045

This article is protected by copyright. All rights reserved. 
Takeichi M (2011) Self-organization of animal tissues: cadherin-mediated processes. Developmental cell 21: $24-26$

Tay HG, Ng YW, Manser E (2010) A vertebrate-specific Chp-PAK-PIX pathway maintains Ecadherin at adherens junctions during zebrafish epiboly. PloS one 5: e10125

Thuault S, Hayashi S, Lagirand-Cantaloube J, Plutoni C, Comunale F, Delattre O, Relaix F, Gauthier-Rouviere C (2013) P-cadherin is a direct PAX3-FOX01A target involved in alveolar rhabdomyosarcoma aggressiveness. Oncogene 32: 1876-1887

Ulrich F, Krieg M, Schotz EM, Link V, Castanon I, Schnabel V, Taubenberger A, Mueller D, Puech PH, Heisenberg CP (2005) Wnt11 functions in gastrulation by controlling cell cohesion through Rab5c and E-cadherin. Developmental cell 9: 555-564

Vassilieva EV, Ivanov AI, Nusrat A (2009) Flotillin-1 stabilizes caveolin-1 in intestinal epithelial cells. Biochem Biophys Res Commun 379: 460-465

von Philipsborn AC, Ferrer-Vaquer A, Rivera-Milla E, Stuermer CA, Malaga-Trillo E (2005) Restricted expression of reggie genes and proteins during early zebrafish development. The Journal of comparative neurology 482: 257-272

Warga RM, Kimmel CB (1990) Cell movements during epiboly and gastrulation in zebrafish. Development 108: 569-580

\section{Figure legends}

\section{Figure 1: Flotillins knock-down impairs epiboly}

A- Flotillin 1 and 2 and actin expression assessed by immunoblotting of protein extracts from control

This article is protected by copyright. All rights reserved. 
embryo (MoCtrl) or Flotillin MO embryos (both MoATGFlot2 and MoSplFlot2). Flotillin 2 knockdown is correlated with a reduction in Flotillin 1 expression, leading us to talk about Flotillins knockdown.

B- Differential interference contrast images of zebrafish embryos at different stages (4-, 16- and 1000cell stage, 75\% epiboly, Prim-6 and 25 hpf) after injection of MoCtrl or MoATGFlot2 oligonucleotides or after rescue by co-injection of mouse Flotillin 2 mRNA with MoATGFlot2 oligonucleotides. Bar $=100 \mu \mathrm{m}$.

C- The graph shows the percentage of embryos that completed a normal epiboly (white), of embryos that were strongly delayed and reached $60-75 \%$ epiboly (orange) and of embryos that were arrested/dead between 50-60\% epiboly (red) at $9.5 \mathrm{hpf}$. The number of embryos analyzed for each condition (from at least three independent experiments) is indicated in between brackets.

D- Representative confocal images of Flotillin 2 (left panels) or Flotillin 1 (right panels) distribution in the dorsal part of zebrafish embryos at the indicated stages. Inserts show the entire embryos. Bar $=$ $30 \mu \mathrm{m}$.

This article is protected by copyright. All rights reserved. 

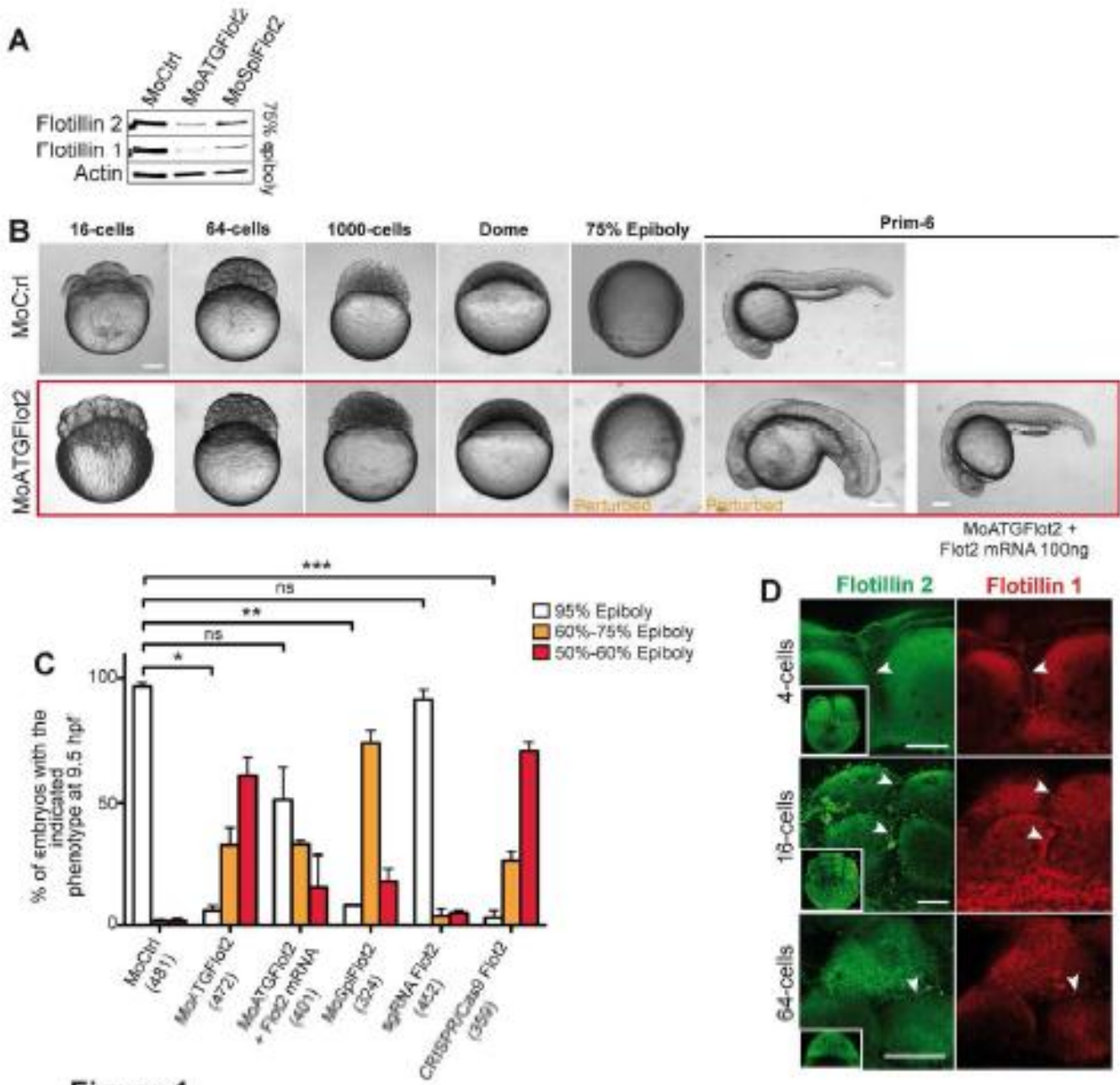

Figure 1

Figure 2: Flotillins knock-down perturbs DC migration and radial cell intercalation during epiboly

A- Confocal images of control (MoCtrl) and Flotillin MO (MoATGFlot2) embryos at $75 \%$ epiboly stained with phalloidin (F-actin) and Hoechst (nuclei). The yellow line measures the distance between the margin of migrating EVL cells and DCs and indicates that DC migration is delayed in Flotillins MOs, whereas EVL migration is not affected. Bar $=30 \mu \mathrm{m}$.

This article is protected by copyright. All rights reserved. 
B- The distance (B) between the DC margin and the F-actin ring in YSL at the EVL margin was measured in control and Flotillin MO embryos. At least 25 embryos were analyzed from three different experiments.

C- DC movement was recorded using time-lapse microscopy in phase contrast acquisitions of one plane of embryos at $75 \%$ epiboly. Shown are representative images of DC movement in control (MoCtrl) and Flotillin MO (MoATGFlot2) embryos. Some cells are colored to follow their movement in the layer during the recording period. Stars indicate a DC inserted in the migrating layer. Bar $=10$ $\mu \mathrm{m}$.

D- Trajectories of DCs in control and Flotillin MOs during 20 min.

E - Directionality measured in DCs in control and Flotillin MOs during 20 min.

F- CCC duration was measured in DCs of control (MoCtrl) and Flotillin MOs (MoATGFlot2) during $20 \mathrm{~min}$.

G- Control (MoCtrl) and Flotillin MO (MoATGFlot2) embryos were co-injected with GFP-CAAXRas to clearly visualize cells and to follow DC movement. Shown are y-z projections of images from a 15-minute time-lapse recording to visualize cell tracking during the recording period. The lines indicate cell trajectories. The graph represents the trajectories of all cells.

H, I, J and K- Boxplots represent the quantification of the total speed (H), instantaneous speed (I), net speed $(\mathrm{J})$ and persistence (K) in control (MoCtrl) and Flotillin MOs (MoATGFlot2) embryos during $30 \mathrm{~min}$.

L- Schematic representation of the different speeds measured in $\mathrm{H}$, I and $\mathrm{J}$.

From $C$ to F: MoCtrl ( $n=34$ cells from $n=7$ embryos) and MoATGFlot2 $(n=54$ cells from $n=10$ embryos) embryos from at least three independent experiments. From $\mathrm{H}$ to $\mathrm{K}$ : MoCtrl $(\mathrm{n}=41$ cells

This article is protected by copyright. All rights reserved. 
Figure 3: Flotillins knock-down impairs E-cadherin and $\beta$-catenin accumulation at cell-cell contacts of DCs

A- Zebrafish embryo injected with a mRNA encoding GFP-tagged Flotillin 2 and stained using an anti-E-cadherin antibody and Hoescht (nuclei). Shown are confocal images of DCs. The co-localized Flotillin 2 and E-cadherin signals were highlighted using the «Colocalization Threshold» tool from Image $\mathrm{J}$ and are shown in white. Pearson's coefficient $=0.64$. Bar $=10 \mu \mathrm{m}$.

B- Analysis of E-cadherin, $\beta$-catenin and F-actin (insets) in DCs at $75 \%$ epiboly in control (MoCtrl) and Flotillin MO (MoATGFlot2) embryos by confocal microscopy. Bar $=10 \mu \mathrm{m}$.

$\mathrm{C}$ and D- Intensity profiles of E-cadherin and $\beta$-catenin signals in control (MoCtrl)(black) and Flotillins MO (MoATGFlot2)(red) embryos were measured along lines (as shown in figure B) by line scanning (ImageJ software).

E, F, and G- Boxplots represent the normalized intensity values of the F-actin, E-cadherin and $\beta$ catenin signals at cell-cell contacts (CCC) in control (MoCtrl) and Flotillins MO (MoATGFlot2) embryos.

H- Quantification of the F-actin, E-cadherin and $\beta$-catenin signal at CCC and outside CCC (NCC). Shown is the CCC signal/NCC signal ratio in control (MoCtrl) and Flotillins MO (MoATGFlot2) embryos.

Panels $E$ to H. For E-cadherin: $n=20$ cells from $n=12$ MoCtrl embryos; $n=16$ cells from $n=10$ MoATGFlot 2 embryos. For $\beta$-catenin: $n=14$ cells from $n=8$ MoCtrl embryos; $n=15$ cells from $n=$ 7 MoATGFlot2 embryos, from four independent experiments.

I- E-cadherin, $\beta$-catenin and actin expression assessed by immunoblotting in protein extracts from control (MoCtrl) and Flotillins MO (MoATGFlot2) embryos (50 embryos were pooled for each condition).

This article is protected by copyright. All rights reserved. 
J- E-cadherin, F $\square \square \square \square \square \square \square \square \square \square$ and actin expression assessed by immunoblotting in protein extracts from control (MoCtrl) and Flotillins MO (MoATGFlot2) embryos treated with DMSO or GM6001 $(100 \mu \mathrm{M})(50$ embryos were pooled for each condition).

For all panels: values are the mean \pm SEM. ns: non-significant; **, $\mathrm{P}<0.05 ; * * *, \mathrm{P}<0.005 ; * * * *, \mathrm{P}$ $<0.0005$.

This article is protected by copyright. All rights reserved. 

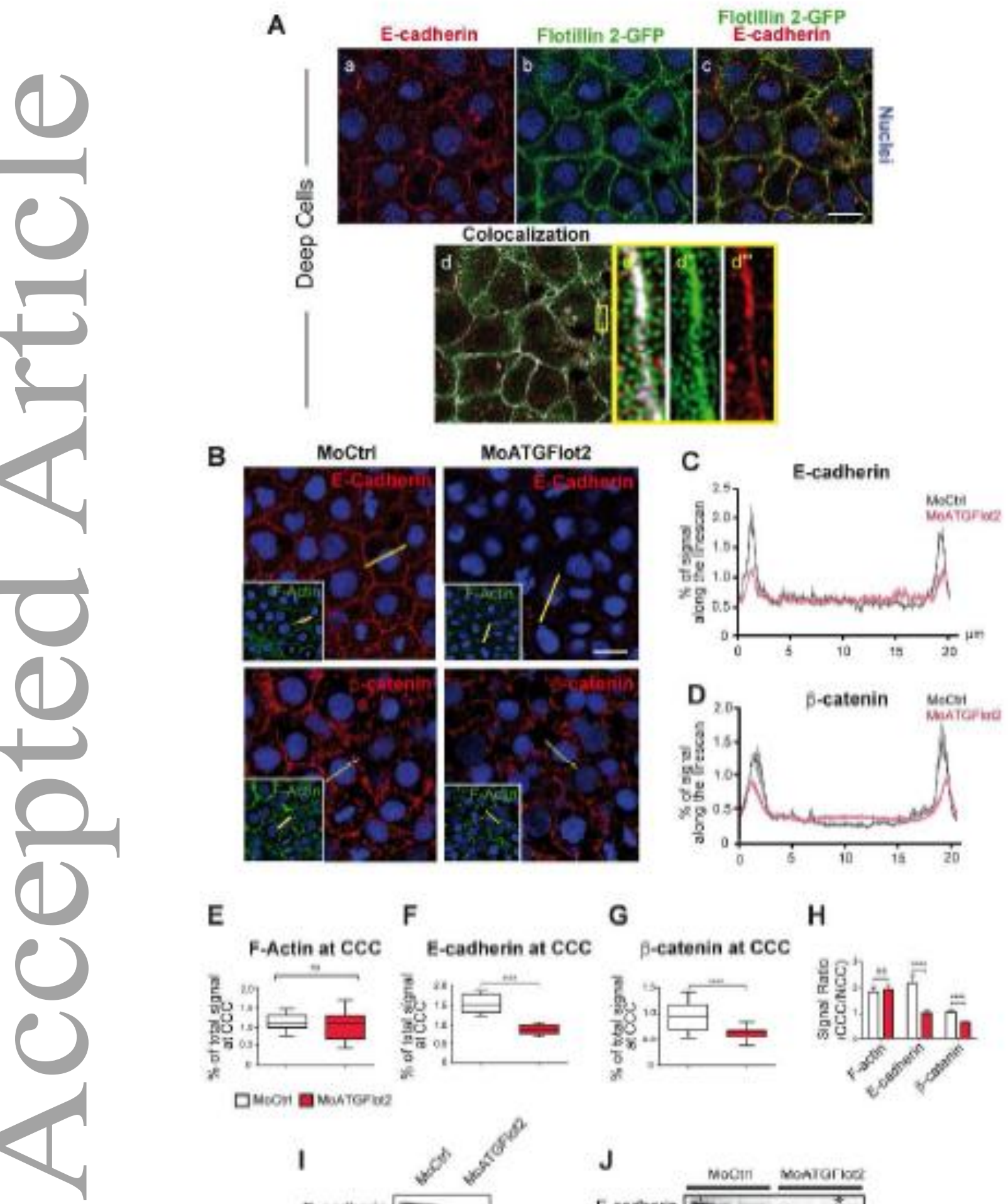

DMUGH DMNTGR

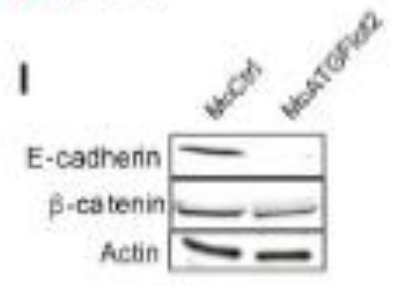

Figure 3

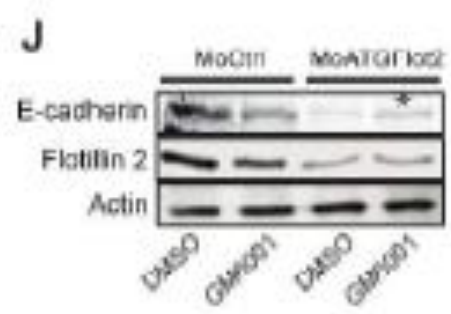

This article is protected by copyright. All rights reserved. 


\section{Figure 4: Model for the role of Flotillins in zebrafish epiboly}

Epiboly is an important morphogenetic process during gastrulation that involves different cell types. At the beginning of gastrulation (left panel) DCs undergo radial cell intercalation (RCI), leading to deeper cell intercalation between and displacement of more superficial cells. This allows the presumptive ectoderm to become thinner and migrate over the yolk ( $75 \%$ epiboly, right-upper panel). Flotillins are required for E-cadherin-mediated cell-cell contact formation that allows the correct mechanical coupling between DCs during epiboly. In Flotillins knock-down embryos, E-cadherinmediated cell-cell contacts are perturbed and DC RCI cannot occurs, thus impairing epiboly completion (right-bottom panel). Although EVL cell morphology and E-cadherin-mediated adhesion are perturbed, these cells appear to undergo normal epiboly.

This article is protected by copyright. All rights reserved. 


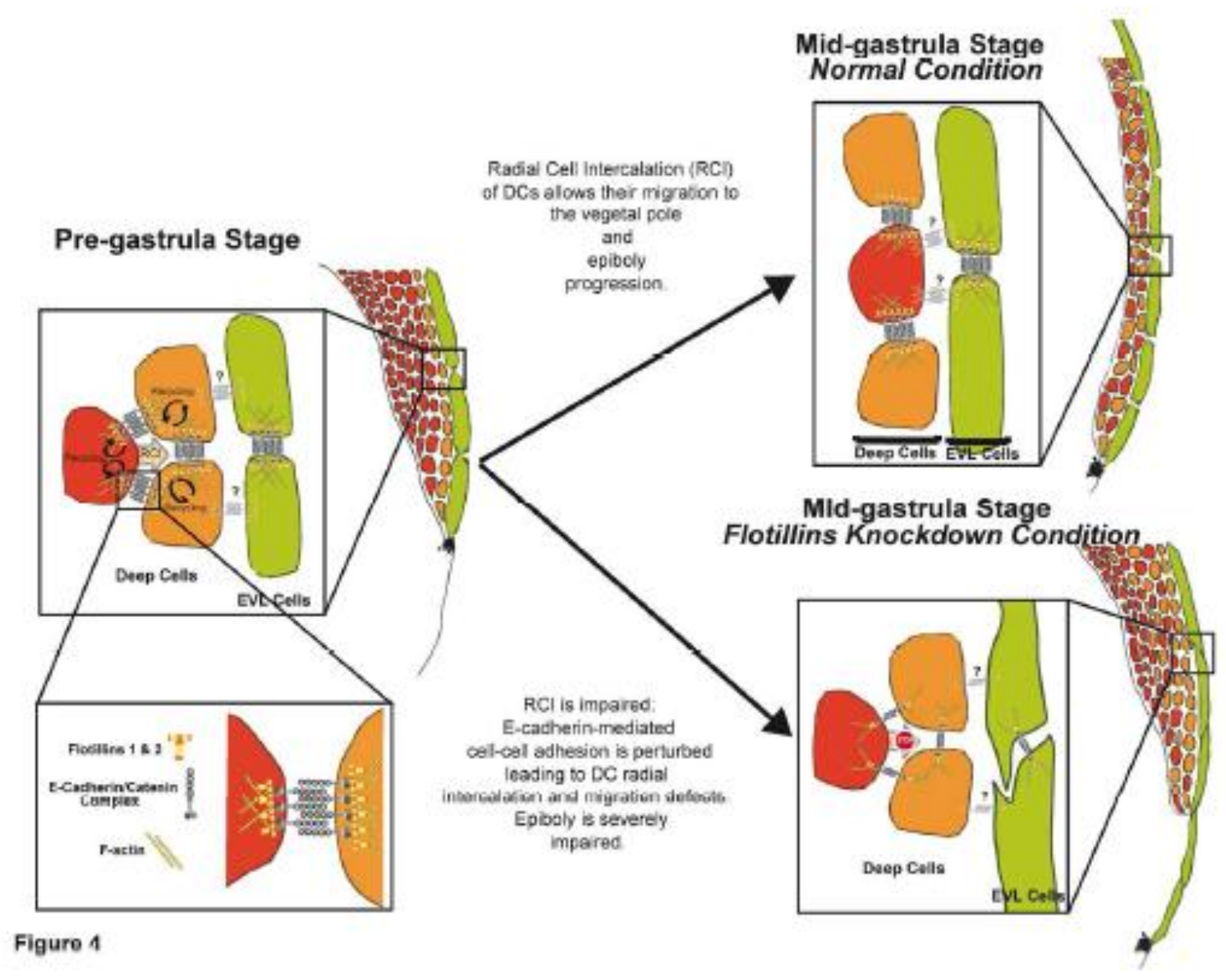

\section{Graphical Abstract}

Collective cell migration, which enables fundamental processes in morphogenesis, tissue repair, and cancer invasion, has long been known to be controlled by cell-cell adhesion molecules of the cadherin family. Using zebrafish and integrated cell biology approaches, we identified Flotillins as major regulator of this process during zebrafish epiboly, the first coordinated cell movement in most vertebrates that marks the onset of gastrulation.

This article is protected by copyright. All rights reserved. 
